UDC 517.54

S. Yu. GraF

\title{
THE SCHWARZIAN DERIVATIVES OF HARMONIC FUNCTIONS AND UNIVALENCE CONDITIONS
}

\begin{abstract}
In the paper we obtain some analogues of Nehari's univalence conditions for sense-preserving functions that are harmonic in the unit disc $\mathbb{D}=\{z \in \mathbb{C}:|z|<1\}$.
\end{abstract}

Key words: harmonic mappings, univalence criteria, Schwarzian derivative

2010 Mathematical Subject Classification: 30C45, 30C55, 30C99

1. Preliminaries. Let $D \subset \mathbb{C}$ be a simply connected domain, $h$ be a locally univalent function, analytic in $D$. The Schwarzian derivative of $h$ is defined (cf., [11, 7]) as

$$
S[h](z)=\left(\frac{h^{\prime \prime}(z)}{h^{\prime}(z)}\right)^{\prime}-\frac{1}{2}\left(\frac{h^{\prime \prime}(z)}{h^{\prime}(z)}\right)^{2} .
$$

An important role of the Schwarzian derivative in theory of univalent analytic functions is well known. Almost 70 years ago Z. Nehari [18] made the following deep observation: let $h$ be a locally univalent analytic function in a simply connected domain and its Schwarzian derivative $S[h]=2 \psi$; then $h$ is univalent iff every non-trivial solution of the differential equation $u^{\prime \prime}+\psi u=0$ has no more than one zero. This key result reduces the univalence problem to the classical Sturm comparison theorem [17. Later [19] Nehari proved

Theorem A. Let $h$ be a locally univalent analytic function in $\mathbb{D}=\{z \in$ $\in \mathbb{C}:|z|<1\}$ and

$$
|S[h](z)| \leq 2 p(|z|) \text { in } \mathbb{D} .
$$

Here the function $p(x)$ (also called a Nehari function) is positive, continuous, even on the interval $(-1,1)$, and has the following properties: 
$\left(1-x^{2}\right)^{2} p(x)$ is nonincreasing on $[0,1)$ and no non-trivial solution of the differential equation $u^{\prime \prime}+p u=0$ has more than one zero on $(-1,1)$.

Then $h$ is globally univalent in $\mathbb{D}$.

The well known special case of this theorem claims univalence of $h$ if $|S[h](z)| \leq 2 /\left(1-|z|^{2}\right)^{2}$ in $\mathbb{D}$.

Theorem A and its special cases encouraged many mathematicians to extend these Nehari's results to different classes of functions. For example, L. Ahlfors and G. Weill 2 established the condition under which a univalent analytic function in $\mathbb{D}$ has a quasiconformal extension onto the whole Riemann sphere. Also, L. Ahlfors [1] defined a version of the Schwarzian derivative that provides injectivity criteria for curves $\gamma:(-1,1) \rightarrow \mathbb{R}^{n}$. Gehring and Pommerenke [9] applied the Schwarzian derivative of analytic functions to study quasicircles.

During the recent decades several attempts to generalize the Schwarzian derivative and Theorem A onto the case of harmonic functions were also made. We remind (cf., [8]) that every sense-preserving function $f(z)$, harmonic in the unit disk $\mathbb{D}$, can be represented as $f(z)=h(z)+\overline{g(z)}$, where $h$ and $g$ are analytic in $\mathbb{D}$. The dilatation $\omega(z)=g^{\prime}(z) / h^{\prime}(z)$ is analytic in $\mathbb{D}$ and $|\omega(z)|<1$ for all $z \in \mathbb{D}$.

In 2003 the Schwarzian derivative was generalized by P. Duren, B. Osgood, and M. Chuaqui [4] to the case of harmonic functions $f=h+\bar{g}$ in the disk $\mathbb{D}$ with the dilatation $\omega=g^{\prime} / h^{\prime}=q^{2}$, where $q$ is some analytic function in $\mathbb{D}$ and $\left|h^{\prime}\right|+\left|g^{\prime}\right|>0$. Their definition is given by

$$
\begin{aligned}
& \mathcal{S}_{f}(z)=2\left(\ln \left(\left|h^{\prime}(z)\right|+\left|g^{\prime}(z)\right|\right)\right)_{z z}-\left(\ln \left(\left|h^{\prime}(z)\right|+\left|g^{\prime}(z)\right|\right)\right)_{z}^{2}= \\
& =S[h](z)+\frac{2 \overline{q(z)}}{1+|q(z)|^{2}}\left(q^{\prime \prime}(z)-q^{\prime}(z) \frac{h^{\prime \prime}(z)}{h^{\prime}(z)}\right)-\left(\frac{2 q^{\prime}(z) \overline{q(z)}}{1+|q(z)|^{2}}\right)^{2},
\end{aligned}
$$

where $S[h]$ is the classical Schwarzian derivative of an analytic locally univalent function $h$. Note that the function $f$ in definition (1) need not be sense-preserving and locally univalent. This definition obviously can be applied to harmonic functions in arbitrary simply connected domains.

Later R. Hernández and M. J. Martín [15] proposed a modified definition of Schwarzian derivative that is valid for the whole family of sense-preserving harmonic mappings. This definition preserves the main 
properties of the classical Schwarzian derivative and is following:

$$
\begin{aligned}
& \mathbb{S}_{f}(z)=\left(\ln \left(\left|h^{\prime}(z)\right|^{2}-\left|g^{\prime}(z)\right|^{2}\right)\right)_{z z}-\frac{1}{2}\left(\ln \left(\left|h^{\prime}(z)\right|^{2}-\left|g^{\prime}(z)\right|^{2}\right)\right)_{z}^{2}= \\
& =S[h](z)+\frac{\overline{\omega(z)}}{1-|\omega(z)|^{2}}\left(\frac{h^{\prime \prime}(z)}{h^{\prime}(z)} \omega^{\prime}(z)-\omega^{\prime \prime}(z)\right)-\frac{3}{2}\left(\frac{\omega^{\prime}(z) \overline{\omega(z)}}{1-|\omega(z)|^{2}}\right)^{2} .
\end{aligned}
$$

Both definitions of Schwarzian derivatives of harmonic functions possess the chain rule property (cf., [15]) exactly in the same form as in the analytic case. Let $f$ be a sense preserving harmonic function, $\varphi$ be a locally univalent analytic function such that the composition $f \circ \varphi$ is defined; then

$$
\begin{aligned}
& \mathcal{S}_{f \circ \varphi}(z)=\mathcal{S}_{f} \circ \varphi(z) \cdot\left(\varphi^{\prime}(z)\right)^{2}+\mathcal{S}_{\varphi}(z), \\
& \mathbb{S}_{f \circ \varphi}(z)=\mathbb{S}_{f} \circ \varphi(z) \cdot\left(\varphi^{\prime}(z)\right)^{2}+\mathbb{S}_{\varphi}(z) .
\end{aligned}
$$

The Schwarzian derivative $\mathbb{S}_{f}$ is also invariant under affine transformations of a harmonic function $f$ : if $A(w)=a w+b \bar{w}+c,|a|>|b|$, then

$$
\mathbb{S}_{A \circ f}(z) \equiv \mathbb{S}_{f}(z) \text {. }
$$

The properties of the Schwarzian derivatives (1), (2) of harmonic functions have been intensively studied in many papers from different points of view. In particular, the authors of [5] observed a deep connection of $\mathcal{S}_{f}$ with lifts of harmonic functions onto minimal surfaces. In [15, 16] some estimations of $\mathbb{S}_{f}$ in some subclasses of univalent harmonic functions were obtained and many properties of the Schwarzian were established. Norms of the Pre-Schrarzian and Schwarzian derivative $\mathbb{S}_{f}$ were estimated in [14] for the linear- and affine-invariant families of harmonic functions in terms of order of the family; so, analogues of the Krauss and Nehari theorem about the upper bounds of $\left|\mathbb{S}_{f}\right|$ were obtained.

The special attention, of course, was paid to the problem of univalence criteria for harmonic functions in terms of their Schwarzian.

Let a harmonic function $f=h+\bar{g}$ have dilatation $\omega=q^{2}$, where $q$ is analytic (or even meromorphic) in $\mathbb{D}$. Then, according to the WeierstrassEnneper formula (see, cf. [8]), the function $f$ lifts locally to a minimal surface $X_{f}$ with the conformal parametrization $\tilde{f}(z)=(u(z), v(z), t(z)), z \in$ $\in \mathbb{D}$, where

$$
u(z)=\operatorname{Ref}(\mathrm{z}), \quad v(z)=\operatorname{Im} \mathrm{f}(\mathrm{z}), \quad t(z)=2 \operatorname{Im} \int_{\mathrm{z}_{0}}^{\mathrm{z}} \mathrm{q}(\zeta) \mathrm{h}^{\prime}(\zeta) \mathrm{d} \zeta .
$$


The first fundamental form of the minimal surface $X_{f}$ is given by $d s^{2}=\lambda^{2}(z)|d z|^{2}$, where $\lambda=\left|h^{\prime}\right|+\left|g^{\prime}\right| ; \lambda^{2}$ is called the conformal factor. It is known that for every univalent harmonic function $f$ of the prescribed form its lift $\tilde{f}$ is also univalent and defines a non-parametric minimal surface. Vice versa, every non-parametric minimal surface $X=$ $=\{u(z), v(z), F(u(z), v(z))\}$ with the conformal parameter $z \in \mathbb{D}$ has a projection $f=u+i v$ that is an univalent harmonic mapping of $\mathbb{D}$; also, representation (5) is unique for $f$ and $X$ up to vertical shifts and reflection relative to the plane $t=0$. The authors of [5] used the Ahlfors generalized Schwarzian for curves in $\mathbb{R}^{3}$ to obtain the following univalence criteria for lifts of a harmonic function $f$ to the minimal surface:

Theorem B. Let $f=h+\bar{g}$ be a harmonic function in $\mathbb{D}$, its dilatation $\omega=q^{2}$ for some meromorphic function $q$, and $\lambda=\left|h^{\prime}\right|+\left|g^{\prime}\right| \neq 0$. Let $\tilde{f}$ be Weierstrass-Enneper lift of $f$ to the minimal surface $X_{f}$ with the Gauss curvature $K(z)$ at a point $\tilde{f}(z)$. Suppose that

$$
\left|\mathcal{S}_{f}(z)\right|-\lambda^{2}(z) K(z) \leq 2 p(|z|) \text { in } \mathbb{D}
$$

for some Nehari function $p$. Then $\tilde{f}$ (and $f$ ) is univalent in $\mathbb{D}$.

The univalence criteria for $f$ itself is a consequence.

Note that the Gauss curvature of the minimal surface is non-positive. If a function $f$ is analytic, then the $X_{f}$ is a plane, $K \equiv 0, \mathcal{S}_{f}=S[f]$, and Theorem B coincides with the classical result of Nehari.

Another univalence condition for sense-preserving harmonic functions $f$ was obtained in terms of Schwarzian derivative $\mathbb{S}_{f}$.

In [16] R. Hernández and M. J. Martín proved an analogue of Theorem A for the $\mathbb{S}_{f}$ in the following form: they proved the existence of constant $C$ such that for $f=h+\bar{g}$ the inequality

$$
\left|\mathbb{S}_{f}(z)\right| \leq \frac{C}{\left(1-|z|^{2}\right)^{2}} \text { for all } z \in \mathbb{D}
$$

implies the univalence of the analytic part $h$ of $f$ and, as a consequence, the global univalence of $f$. However, the constant $C$ was not estimated.

In this paper we give analogues of Theorems A and B in terms of the Schwarzian derivative $\mathbb{S}_{f}$ for an arbitrary sense-preserving harmonic function $f$ in $\mathbb{D}$.

2. Univalence conditions for harmonic functions. It is convenient to assume in the sequel that a harmonic function $f=h+\bar{g}$ is 
normalized: $f(0)=0, h^{\prime}(0)=1$. It is clear that this normalization does not influence on univalence of $f$ nor on the values of Schwarzian derivatives.

First we consider a harmonic sense-preserving function $f=h+\bar{g}$ in $\mathbb{D}$ whose dilatation $\omega$ equals the square of an analytic function $q$ such that $|q(z)|<1$ for all $z \in \mathbb{D}$. Let $\alpha$ be the order ord (f) of the function $f$ (cf., [20, 21]), i.e.,

$$
\alpha:=\operatorname{ord}(\mathrm{f})=\frac{1}{2} \sup _{\mathrm{z} \in \mathbb{D}}\left|\frac{\mathrm{h}^{\prime \prime}(\mathrm{z})}{\mathrm{h}^{\prime}(\mathrm{z})}\left(1-|\mathrm{z}|^{2}\right)-2 \overline{\mathrm{z}}\right| .
$$

This means that $\alpha$ is equal to the supremum of the absolute values of the second coefficients of analytic parts of the functions over the linear invariant family $\mathcal{L}(f)$. This family consists of functions

$$
F(z)=\frac{f(\Phi(z))-f(\Phi(0))}{h^{\prime}(\Phi(0)) \Phi^{\prime}(0)}
$$

where $\Phi(z)=\left(z+z_{0}\right) /\left(1+\overline{z_{0}} z\right)$ and $z_{0}$ runs over the disk $\mathbb{D}$. Properties of the linear and affine invariant families of harmonic functions can be found in $[21,22,12]$.

Note that the order of an univalent analytic or univalent sense preserving harmonic function is always finite (cf., 6, 8]). So, it is natural to assume that $\alpha<\infty$.

Theorem 1. Let a harmonic function $f$ be sense-preserving in $\mathbb{D}, f(0)=$ $=h^{\prime}(0)-1=0$ and $\omega=q^{2}$ in $\mathbb{D}$. Let $\alpha$ be the order of $f$. Then for any $z \in \mathbb{D}$

$$
\left|\mathbb{S}_{f}(z)-\mathcal{S}_{f}(z)\right|<\frac{2 \alpha+7 / 2}{\left(1-|z|^{2}\right)^{2}} .
$$

This estimation is sharp in the sense of the order of growth with $|z| \rightarrow 1-$.

Proof. Let $f$ meet the conditions of Theorem 1. First note that due to the chain rule (3) the difference of the Schwarzian derivatives (2) and (1) at an arbitrary point $z \in \mathbb{D}$ can be expressed in the form

$$
\mathbb{S}_{f}(z)-\mathcal{S}_{f}(z)=\frac{\mathbb{S}_{F}(0)-\mathcal{S}_{F}(0)}{\left(1-|z|^{2}\right)^{2}}
$$

where $F$ has the form $(6), \Phi(\zeta)=(\zeta+z) /(1+\bar{z} \zeta)$, and $\mathbb{S}_{\Phi}=\mathcal{S}_{\Phi}=S[\Phi] \equiv$ $\equiv 0$. Note that the dilatation of the function $F$ has the form $\Omega=e^{i \theta} \omega \circ \Phi=$ 
$=e^{i \theta}(q \circ \Phi)^{2}$ with some constant $\theta \in \mathbb{R}$; so $\Omega=Q^{2}$, i.e., is the square of an analytic function.

The harmonic function $F$ has a representation $F=H+\bar{G}$ with analytic $H$ and $G$. Also, we can assume that $A_{1}=H^{\prime}(0)=1$, because the Schwarzian derivatives are invariant with respect to multiplication on a constant. Then $(Q(0))^{2}=G^{\prime}(0)=B_{1},\left|B_{1}\right|<1$. If $B_{1}=0$, then $\mathbb{S}_{F}(0)=$ $=\mathcal{S}_{F}(0)=S[H](0)$ and $\mathbb{S}_{F}(0)-\mathcal{S}_{F}(0)=0$. So, we assume that $\left|B_{1}\right| \in$ $\in(0,1)$.

Now we express the difference $\mathbb{S}_{F}(0)-\mathcal{S}_{F}(0)$ in terms of coefficients $B_{1}$ and $A_{2}=H^{\prime \prime}(0) / 2$ of the function $F$. By a straightforward though rather bulky calculations it is possible to show that

$$
\begin{aligned}
& \left|\mathbb{S}_{F}(0)-\mathcal{S}_{F}(0)\right|=\frac{2|Q(0)|}{1-|Q(0)|^{4}} \times \\
& \times\left|\frac{H^{\prime \prime}(0)}{H^{\prime}(0)} Q^{\prime}(0)-Q^{\prime \prime}(0)+\frac{\overline{Q(0)}\left(Q^{\prime}(0)\right)^{2}}{1-|Q(0)|^{4}}\left(1-4|Q(0)|^{2}\right)\right| \leq \frac{2 \sqrt{\left|B_{1}\right|}}{1-\left|B_{1}\right|^{2}} \times \\
& \times\left\{2\left|A_{2} Q^{\prime}(0)\right|+\left|Q^{\prime \prime}(0)\right|+\sqrt{\left|B_{1}\right|}\left(\frac{\left|Q^{\prime}(0)\right|}{1-\left|B_{1}\right|}\right)^{2} \frac{1-\left|B_{1}\right|}{1+\left|B_{1}\right|}|1-4| B_{1}||\right\} .
\end{aligned}
$$

The analytic in $\mathbb{D}$ function $Q$ meets the conditions of the well-known Schwarz Lemma (cf., [1] $)$. So, we can estimate its derivatives at the origin:

$$
\begin{aligned}
& \left|Q^{\prime}(0)\right| \leq 1-|Q(0)|^{2}=1-\left|B_{1}\right|<1, \\
& \left|Q^{\prime \prime}(0)\right| \leq 2\left(1-|Q(0)|^{2}\right)=2\left(1-\left|B_{1}\right|\right)<2 .
\end{aligned}
$$

Therefore, due to (8), we obtain an estimation

$$
\left|\mathbb{S}_{F}(0)-\mathcal{S}_{F}(0)\right|<\frac{2 \sqrt{\left|B_{1}\right|}}{1+\left|B_{1}\right|}\left\{2\left|A_{2}\right|+2+\sqrt{\left|B_{1}\right|} \frac{|1-4| B_{1}||}{1+\left|B_{1}\right|}\right\} .
$$

It is easy to see that for $x \in(0,1)$ both functions $x /\left(1+x^{2}\right)$ and $x \mid 1-$ $-4 x^{2} \mid /\left(1+x^{2}\right)$ tend to their suprema when $x \rightarrow 1-$. Then

$$
\left|\mathbb{S}_{F}(0)-\mathcal{S}_{F}(0)\right|<2\left|A_{2}\right|+2+\frac{3}{2} .
$$

To finish the proof note that

$$
A_{2}=\frac{1}{2}\left(\frac{h^{\prime \prime}(z)}{h^{\prime}(z)}\left(1-|z|^{2}\right)-2 \bar{z}\right),
$$


so $\left|A_{2}\right| \leq \alpha$ when $z$ runs over the disk $\mathbb{D}$. Combining the last estimations, we obtain the desired inequality (7).

To illustrate the sharpness of estimation (7), let us construct the harmonic univalent function $f_{0}=h_{0}+\overline{g_{0}}$ with the properties

$$
\begin{aligned}
& g_{0}^{\prime}(z)=z^{2} h_{0}^{\prime}(z), \\
& h_{0}^{\prime}(z)-g_{0}^{\prime}(z)=k^{\prime}(z),
\end{aligned}
$$

where $k(z)=z /(1-z)^{2}$ is the Köebe function, which is univalent in $\mathbb{D}$. Then

$$
h_{0}^{\prime}(z)=\frac{1}{(1-z)^{4}} \text { and } f_{0}(z)=\frac{1 / 3}{(1-z)^{3}}+\frac{\overline{z^{2}-z+1 / 3}}{(1-z)^{3}}-\frac{2}{3} .
$$

The univalence of the function $f_{0}$ is provided by the clever "shear construction" introduced by J. Clunie and T. Sheil-Small (see [6]). Even more, the range of $\mathbb{D}$ under the mapping $f_{0}$ is convex in the horizontal direction, i.e., $f_{0}(\mathbb{D})$ has connected (or empty) intersections with any horizontal line in $\mathbb{C}$. The direct calculations show that

$$
\mathbb{S}_{f_{0}}(z)-\mathcal{S}_{f_{0}}(z)=\frac{2 \bar{z}}{1-|z|^{4}}\left(\frac{4}{1-z}+\frac{\bar{z}\left(1-4|z|^{2}\right)}{1-|z|^{4}}\right) .
$$

For $z=x \in(-1,1)$ obtain

$$
\mathbb{S}_{f_{0}}(x)-\mathcal{S}_{f_{0}}(x)=\frac{2 x\left(4 x^{2}+5 x+4\right)}{\left(1-x^{2}\right)^{2}\left(1+x^{2}\right)^{2}} \approx \frac{13}{2} \frac{1}{\left(1-x^{2}\right)^{2}}
$$

when $x$ tends to 1 . So, the order of growth in (7) is sharp.

The proved estimation (7) allows us to apply Theorem B to the Schwarzian derivative $\mathbb{S}_{f}$ and to obtain the corresponding univalence condition. Further in this paper we assume that $f$ is not analytic.

Theorem 2. Let a harmonic function $f$ be sense-preserving in $\mathbb{D}$ with the dilatation $\omega=q^{2}$ in $\mathbb{D}$ and $\alpha<\infty$ be an order of $f$. Let $\tilde{f}$ be the lift (5) of the mapping $f$ to a minimal surface and assume that inequality

$$
\left|\mathbb{S}_{f}(z)\right|+\frac{2 \alpha+15 / 2}{\left(1-|z|^{2}\right)^{2}} \leq 2 p(|z|)
$$

holds for some Nehari function $p$ for all $z \in \mathbb{D}$. Then $\tilde{f}$ and $f$ are univalent in $\mathbb{D}$. 
Proof. Let conditions of the theorem be fulfilled. Denote the minimal surface determined by the lift $\tilde{f}$ of the function $f$ by $X_{f}$ and its curvature by $K(z)$. Then apply inequality (7) to obtain

$$
\left|\mathcal{S}_{f}(z)\right|-\lambda^{2}(z) K(z)<\left|\mathbb{S}_{f}(z)\right|+\frac{2 \alpha+7 / 2}{\left(1-|z|^{2}\right)^{2}}-\lambda^{2}(z) K(z)
$$

for all $z \in \mathbb{D}$. This implies univalence of $\tilde{f}$ and $f$ provided that there exists a Nehari function $p(x)$ such that $(9)$ is dominated by $2 p(|z|)$. In order to finish the proof we need to estimate the term $\lambda^{2}(z) K(z)$ in (9). Indeed, the Gauss curvature of the minimal surface $X_{f}$ has the form (see [8])

$$
K(z)=-4 \frac{\left|q^{\prime}(z)\right|^{2}}{\left|h^{\prime}(z)\right|^{2}\left(1+|q(z)|^{2}\right)^{4}} .
$$

Therefore,

$$
\begin{aligned}
-\lambda^{2}(z) K(z) & =4 \frac{\left|q^{\prime}(z)\right|^{2}\left(\left|h^{\prime}(z)\right|+\left|g^{\prime}(z)\right|\right)^{2}}{\left|h^{\prime}(z)\right|^{2}\left(1+|q(z)|^{2}\right)^{4}}=4 \frac{\left|q^{\prime}(z)\right|^{2}}{\left(1+|q(z)|^{2}\right)^{2}} \leq \\
& \leq \frac{4}{\left(1-|z|^{2}\right)^{2}}\left(\frac{1-|q(z)|^{2}}{1+|q(z)|^{2}}\right)^{2} \leq \frac{4}{\left(1-|z|^{2}\right)^{2}}
\end{aligned}
$$

because $\left|q^{\prime}(z)\right| \leq\left(1-|q(z)|^{2}\right) /\left(1-|z|^{2}\right)$ due to the Schwarz Lemma. Combine the last inequality with (9) and apply Theorem B to obtain the desired conclusion of the theorem.

Note that estimation of the quantity $\lambda^{2} K$ used in the proof above is sharp. So, the condition on $\mathbb{S}_{f}$ in Theorem 2 can not be weakened in a general case in the sense of the order of growth.

Now we are going to show that the analogue of the statement about univalence of $f$ in Theorem 2 is still valid without any assumption about the dilatation of $f$.

Theorem 3. Let a harmonic function $f$ be sense-preserving in $\mathbb{D}, f(0)=$ $=h^{\prime}(0)-1=0, \alpha<\infty$ be an order of $f$ and

$$
\left|\mathbb{S}_{f}(z)\right|+\frac{2 \alpha+19 / 2}{\left(1-|z|^{2}\right)^{2}}<2 p(|z|)
$$

for some Nehari function $p$ and for all $z \in \mathbb{D}$. Then $f$ is univalent in $\mathbb{D}$. 
Proof. Let the conditions of the theorem are fulfilled and $\omega=g^{\prime} / h^{\prime}$ be a dilatation of $f$. It is convenient to assume here that $f(0)=h^{\prime}(0)-1=$ $=0$. As was have remarked above, this assumption does not influence on univalence of $f$ or on the value of its Schwarzian derivatives.

Suppose that $\omega$ can not be represented as a square of an analytic function in $\mathbb{D}$. Therefore, $\omega$ has zeros in $\mathbb{D}$. Fix an arbitrary $\rho \in(0,1)$ and define a harmonic sense-preserving function

$$
f_{\rho}(z)=\frac{1}{\rho} f(\rho z)
$$

The univalence of $f_{\rho}$ in $\mathbb{D}$ is equivalent to that of $f$ in the disk $|z|<\rho$.

Consider a positive $\varepsilon \in(0,1)$ and define an affine deformation of $f_{\rho}$ :

$$
f_{\rho, \varepsilon}(z)=\frac{f(\rho z)+\varepsilon \overline{f(\rho z)}}{\rho\left(1+\varepsilon g^{\prime}(0)\right)} .
$$

Note that $f_{\rho}$ and $f_{\rho, \varepsilon}$ are univalent (or opposite) simultaneously. The dilatation of $f_{\rho, \varepsilon}$ has the form

$$
\omega_{\rho, \varepsilon}(z)=e^{i \theta} \frac{\omega(\rho z)+\varepsilon}{1+\varepsilon \omega(\rho z)}, \theta \in \mathbb{R}
$$

Note that $|\omega(\rho z)|<(\rho+|\omega(0)|) /(1+\rho|\omega(0)|)$ for all $z \in \mathbb{D}$ : this is a simple consequence of the Schwarz Lemma. Let us choose a $\varepsilon$ such that

$$
\frac{\rho+|\omega(0)|}{1+\rho|\omega(0)|}<\varepsilon<1 .
$$

Then $\omega_{\rho, \varepsilon}$ does not have zeros in $\mathbb{D}$ and, therefore, there exists an analytic $q$ such that $q^{2}=\omega_{\rho, \varepsilon}$.

Now show that condition (10) allows to apply Theorem B to the functions $f_{\rho, \varepsilon}$ for any arbitrary $\rho<1$ and the corresponding $\varepsilon$. For this purpose, transform the proofs of Theorems 1, 2 to obtain an estimation for

$$
\left|\mathcal{S}_{f_{\rho, \varepsilon}}(z)\right|-\lambda_{\rho, \varepsilon}^{2}(z) K_{\rho, \varepsilon}(z),
$$

where $\lambda_{\rho, \varepsilon}^{2}$ and $K_{\rho, \varepsilon}$ are the conformal factor and the Gauss curvature, respectively, of the minimal surface that corresponds to the function $f_{\rho, \varepsilon}$.

First note that

$$
\mathbb{S}_{f_{\rho, \varepsilon}}(z)=\mathbb{S}_{f_{\rho}}(z)
$$


due to the affine invariance (4) of $\mathbb{S}_{f}$. Direct calculations show that

$$
\begin{aligned}
\mathbb{S}_{f_{\rho}}(z) & =\left(\ln \left(\left|(h(\rho z))^{\prime}\right|^{2}-\left|(g(\rho z))^{\prime}\right|^{2}\right)\right)_{z z}- \\
& -\frac{1}{2}\left(\ln \left(\left|(h(\rho z))^{\prime}\right|^{2}-\left|(g(\rho z))^{\prime}\right|^{2}\right)\right)_{z}^{2}=\rho^{2} \mathbb{S}_{f}(\rho z)
\end{aligned}
$$

and, therefore

$$
\mathbb{S}_{f_{\rho, \varepsilon}}(z)=\rho^{2} \mathbb{S}_{f}(\rho z) .
$$

Apply the the chain rule (3) to the Schwarzian derivatives of the function $F_{\rho, \varepsilon}$ (obtained by $(6)$ from $f_{\rho, \varepsilon}$ ), similarly to the proof of Theorem 1 , to derive the estimation

$$
\left|\mathcal{S}_{f_{\rho, \varepsilon}}(z)-\mathbb{S}_{f_{\rho, \varepsilon}}(z)\right|=\frac{\left|\mathcal{S}_{f_{\rho, \varepsilon}}(0)-\mathbb{S}_{f_{\rho, \varepsilon}}(0)\right|}{\left(1-|z|^{2}\right)^{2}}<\frac{2\left|A_{2}(\rho, \varepsilon)\right|+\frac{7}{2}}{\left(1-|z|^{2}\right)^{2}} .
$$

Here $A_{2}(\rho, \varepsilon)=\left(H_{\rho, \varepsilon}\right)^{\prime \prime}(0) / 2$ and $H_{\rho, \varepsilon}$ is the analytic part of the harmonic function $F_{\rho, \varepsilon}$. However, this function belongs to the affine and linear hull of function $f_{\rho}(z)$. The estimation

$$
\operatorname{ord}(\mathcal{A L}) \leq \operatorname{ord}(\mathcal{L})+1
$$

is proved in 13 for the order $\tilde{\alpha}$ of the affine hull $\mathcal{A L}$ of any linear invariant family $\mathcal{L}$. Therefore, $\left|A_{2}(\rho, \varepsilon)\right| \leq \alpha(\rho)+1$. Here $\alpha(\rho)$ denotes order of the harmonic function $f_{\rho}(z)$. In paper [3] D. Campbell proved that

$$
\alpha(\rho) \leq(\alpha-1) \rho+1 \text {. }
$$

The sharp estimation of $\alpha(\rho)$ was obtained in [10, but for our purposes the compact expression cited above is enough. It is clear that $\alpha(\rho) \rightarrow \alpha$ when $\rho$ tends to 1 . As a result, we have

$$
\left|\mathcal{S}_{f_{\rho, \varepsilon}}(z)-\mathbb{S}_{f_{\rho, \varepsilon}}(z)\right|<\frac{2 \alpha+2(\alpha-1)(\rho-1)+\frac{11}{2}}{\left(1-|z|^{2}\right)^{2}} .
$$

Next, obtain

$$
-\lambda_{\rho, \varepsilon}^{2}(z) K_{\rho, \varepsilon}(z) \leq \frac{4}{\left(1-|z|^{2}\right)^{2}}
$$

similarly to the proof of Theorem 2 .

Finally, combining the two last estimations with equality (11), conclude from the condition of Theorem 3 that

$$
\left|\mathcal{S}_{f_{\rho, \varepsilon}}(z)\right|-\lambda_{\rho, \varepsilon}^{2}(z) K_{\rho, \varepsilon}(z)<\rho^{2}\left|\mathbb{S}_{f}(\rho z)\right|+\frac{2 \alpha+2(\alpha-1)(\rho-1)+4+\frac{11}{2}}{\left(1-|z|^{2}\right)^{2}} .
$$


In accordance with the assumption of the theorem

$$
\left|\mathbb{S}_{f}(z)\right|+\frac{2 \alpha+\frac{19}{2}}{\left(1-|z|^{2}\right)^{2}}<2 p(|z|)
$$

for any $z \in \mathbb{D}$. Let $\rho_{1}<1$ be fixed. Continuity of $\mathbb{S}_{f}$ and $p$ implies existence of a $\delta>0$ such that

$$
\left|\mathbb{S}_{f}(z)\right|+\frac{2 \alpha+\frac{19}{2}}{\left(1-|z|^{2}\right)^{2}}<2 p(|z|)-\delta
$$

for any $|z| \leq \rho_{1}$. Therefore,

$$
\begin{aligned}
& \left|\mathcal{S}_{f_{\rho, \varepsilon}}(z)\right|-\lambda_{\rho, \varepsilon}^{2}(z) K_{\rho, \varepsilon}(z)< \\
& <\left|\mathbb{S}_{f}(z)\right|+\frac{2 \alpha+\frac{19}{2}}{\left(1-|z|^{2}\right)^{2}}+\rho^{2}\left|\mathbb{S}_{f}(\rho z)\right|-\left|\mathbb{S}_{f}(z)\right|+\frac{2(\alpha-1)(\rho-1)}{\left(1-|z|^{2}\right)^{2}} \leq \\
& \leq 2 p(|z|)-\delta+\rho^{2}\left|\mathbb{S}_{f}(\rho z)\right|-\left|\mathbb{S}_{f}(z)\right|+\frac{2(\alpha-1)(\rho-1)}{\left(1-|z|^{2}\right)^{2}}
\end{aligned}
$$

Here the last fraction and the difference $\rho^{2}\left|\mathbb{S}_{f}(\rho z)\right|-\left|\mathbb{S}_{f}(z)\right|$ tend to 0 uniformly in $|z| \leq \rho_{1}$ as $\rho \rightarrow 1-$ (and, thus, $\varepsilon \rightarrow 1-$ ). So,

$$
\rho^{2}\left|\mathbb{S}_{f}(\rho z)\right|-\left|\mathbb{S}_{f}(z)\right|+\frac{2(\alpha-1)(\rho-1)}{\left(1-|z|^{2}\right)^{2}}<\delta
$$

for the appropriately chosen $\rho$ that is sufficiently close to 1 . Finally we have

$$
\left|\mathcal{S}_{f_{\rho, \varepsilon}}(z)\right|-\lambda_{\rho, \varepsilon}^{2}(z) K_{\rho, \varepsilon}(z)<2 p(|z|)
$$

for $|z|<\rho_{1}$ if $\rho_{1}<1$ is fixed and $\rho$ and $\varepsilon$ are sufficiently close to 1 .

Here we have to note that if $p(x)$ is a Nehari function then $\tilde{p}(x)=$ $=\rho_{1}^{2} p\left(\rho_{1} x\right)$ is also a Nehari function. Indeed, $\tilde{p}$ is even and $\left(1-x^{2}\right)^{2} \tilde{p}(x)$ is nonincreasing, because

$$
\left(1-x^{2}\right)^{2} \tilde{p}(x)=\rho_{1}^{2} \frac{\left(1-x^{2}\right)^{2}}{\left(1-\rho_{1} x^{2}\right)^{2}}\left(1-\rho_{1} x^{2}\right)^{2} p\left(\rho_{1} x\right),
$$

where $\left(1-\rho_{1} x^{2}\right)^{2} p\left(\rho_{1} x\right)$ is nonincreasing, as well as $\left(1-x^{2}\right) /\left(1-\rho_{1} x^{2}\right)$ for $\rho_{1}<1$.

It is easy to check that if $u$ is a solution of the differential equation

$$
u^{\prime \prime}(x)+p(x) u(x)=0
$$


then the function $\tilde{u}(x)=u\left(\rho_{1} x\right)$ is a solution of

$$
u^{\prime \prime}(x)+\tilde{p}(x) u(x)=0 .
$$

Therefore, if $u_{1}$ and $u_{2}$ are two linear independent solutions of (13), then $\tilde{u}_{1}$ and $\tilde{u}_{2}$ are two linear independent solutions of (14).

No nontrivial linear combination $c_{1} \tilde{u}_{1}(x)+c_{2} \tilde{u}_{2}(x)=c_{1} u_{1}\left(\rho_{1} x\right)+$ $+c_{2} u_{2}\left(\rho_{1} x\right)$ has more than one zero, because $p$ is a Nehari function.

Thus, $\tilde{p}$ is also a Nehari function.

So, if (12) holds for a function $f_{\rho, \varepsilon}$ in $|z| \leq \rho_{1}$, then for $\tilde{f}_{\rho, \varepsilon}=f_{\rho, \varepsilon}\left(\rho_{1} z\right)$ we have

$$
\begin{aligned}
& \left|\mathcal{S}_{\tilde{f}_{\rho, \varepsilon}}(z)\right|-\tilde{\lambda}_{\rho, \varepsilon}^{2}(z) \tilde{K}_{\rho, \varepsilon}(z)= \\
& =\rho_{1}^{2}\left(\left|\mathcal{S}_{f_{\rho, \varepsilon}}\left(\rho_{1} z\right)\right|-\lambda_{\rho, \varepsilon}^{2}\left(\rho_{1} z\right) K_{\rho, \varepsilon}\left(\rho_{1} z\right)\right)<\rho_{1}^{2} 2 p\left(\rho_{1}|z|\right) .
\end{aligned}
$$

Here $\tilde{\lambda}_{\rho, \varepsilon}^{2}(z)=\rho_{1}^{2} \lambda_{\rho, \varepsilon}^{2}\left(\rho_{1} z\right)$ and $\tilde{K}_{\rho, \varepsilon}(z)=K_{\rho, \varepsilon}\left(\rho_{1} z\right)$ (checked by direct calculations).

Therefore,

$$
\left|\mathcal{S}_{\tilde{f}_{\rho, \varepsilon}}(z)\right|-\tilde{\lambda}_{\rho, \varepsilon}^{2}(z) \tilde{K}_{\rho, \varepsilon}(z)<2 \tilde{p}(|z|)
$$

in $|z|<1$ for a Nehari function $\tilde{p}$. From Theorem B we deduce that the function $\tilde{f}_{\rho, \varepsilon}$ is univalent in $\mathbb{D}$ and $f_{\rho, \varepsilon}$ is univalent in a subdisk $|z|<\rho_{1}$. Due to this, $f$ is univalent in the subdisk $|z|<\rho \rho_{1}$. If $\rho_{1} \rightarrow 1-$, then $\rho$ also tends to 1 and $f$ is univalent in $\mathbb{D}$. The theorem is proved.

As the conclusion, let us assume that a harmonic function $f$ is quasiconformal. Then the following version of Theorem 2 is true:

Theorem 4. Let a harmonic function $f$ be sense-preserving in $\underset{\sim}{\mathbb{D}}$ and have finite order, dilatation $\omega=q^{2}$, and $|q(z)| \leq \delta<1$ in $\mathbb{D}$. Let $\tilde{f}$ be a lift (5) of the mapping $f$ to the minimal surface. Then some continuous non-negative function $C(\delta)$ exists, such that $C(0)=0$, and $\tilde{f}$ and $f$ are univalent in $\mathbb{D}$ provided that the inequality

$$
\left|\mathbb{S}_{f}(z)\right|+\frac{C(\delta)}{\left(1-|z|^{2}\right)^{2}} \leq 2 p(|z|)
$$

holds for some Nehari function $p$. In particular, this condition gives the Nehari Theorem $A$ when $\delta \rightarrow 0+$ for functions of finite order.

Indeed, if $|q(z)| \leq \delta$ in $\mathbb{D}$, then dilatation of every function $F$ of the form (6) has the form $Q^{2}$ and $|Q(z)| \leq \delta$. In particular, $\sqrt{\left|B_{1}\right|}=|Q(0)| \leq$ 
$\leq \delta$ and the upper bound in (8) has the form

$$
\left|\mathbb{S}_{F}(0)-\mathcal{S}_{F}(0)\right|<\frac{2 \sqrt{\left|B_{1}\right|}}{1+\left|B_{1}\right|}\left\{2\left|A_{2}\right|+2+\sqrt{\left|B_{1}\right|} \frac{|1-4| B_{1}||}{1+\left|B_{1}\right|}\right\} \leq 2 \delta C_{1}(\delta)
$$

where $C_{1}(\delta)$ is some continuous bounded function on $(0,1)$. An explicit expression for $C_{1}$ can be found by means of symbolic mathematical software; however, as long as $\left|A_{2}\right| \leq \alpha,\left|B_{1}\right| \leq \delta^{2}<1$, it is evident that $C_{1}(\delta) \leq 2 \alpha+7 / 2$, where $\alpha$ is the order of $f$.

Apply the Schwarz Lemma to the function $q / \delta$ to conclude that

$$
\left|q^{\prime}(z)\right| \leq \delta \frac{1-|q(z) / \delta|^{2}}{1-|z|^{2}} \leq \frac{\delta}{1-|z|^{2}}
$$

Therefore, the upper bound of $-\lambda^{2}(z) K(z)$ in the proof of Theorem 2 can be rewritten in the form

$$
\begin{aligned}
& -\lambda^{2}(z) K(z)=4 \frac{\left|q^{\prime}(z)\right|^{2}}{\left(1+|q(z)|^{2}\right)^{2}} \leq \\
& \leq \frac{4 \delta^{2}}{\left(1-|z|^{2}\right)^{2}}\left(\frac{1-|q(z) / \delta|^{2}}{1+|q(z)|^{2}}\right)^{2} \leq \frac{4 \delta^{2}}{\left(1-|z|^{2}\right)^{2}}
\end{aligned}
$$

that tends to 0 when $\delta \rightarrow 0+$.

Introduce a continuous non-negative function $C(\delta)=2 \delta C_{1}(\delta)+4 \delta^{2}$. From above it is clear that

$$
C(\delta) \leq C(\delta) \leq 2 \delta(2 \alpha+7 / 2+2 \delta)
$$

so $C(\delta)$ tends to 0 as $\delta \rightarrow 0+$. Assume that a Nehari function $p$ exists, such that inequality (15) holds in $\mathbb{D}$. Then, repeating actions of the proof of Theorem 2, conclude that

$$
\left|\mathcal{S}_{f}(z)\right|-\lambda^{2}(z) K(z)<\left|\mathbb{S}_{f}(z)\right|+\frac{C(\delta)}{\left(1-|z|^{2}\right)^{2}} \leq 2 p(|z|) .
$$

This inequality and Theorem B provide univalence of the functions $\tilde{f}$ and $f$ in $\mathbb{D}$.

In particular, univalence of $f$ is guaranteed by the inequality

$$
\left|\mathbb{S}_{f}(z)\right| \leq \frac{2-\delta(2 \alpha+7 / 2+4 \delta)}{\left(1-|z|^{2}\right)^{2}}
$$


and (16), provided that $p(x)=1 /\left(1-x^{2}\right)^{2}$ and $\delta$ is small enough. If, in addition, $\delta \rightarrow 0+$, then the quasiconformal harmonic mapping $f$ tends to some analytic function, and Theorem 4 coincides with Theorem A for functions of finite order.

Acknowledgment. The author expresses gratitude to the referees for careful reading of the manuscript and for valuable comments that allowed to improve the paper.

This work is supported by the Russian Science Foundation under grant 17-11-01229.

\section{References}

[1] Ahlfors L. Cross-ratios and Schwarzian derivatives in $\mathbb{R}^{n}$. Complex Analysis: Articles Dedicated to Albert Pfluger on the Occasion of his 80th Birthday, Hersch J. and Huber A., Eds., 1989, Birkhäuser Verlag, Basel, pp. 1-15. DOI: 10.1007/978-3-0348-9158-5.

[2] Ahlfors L., Weill G. A uniqueness theorem for Beltrami equations. Proc. Amer. Math. Soc., 1962, vol. 13, pp. 1975-1978.

[3] Campbell D. M. Locally univalent functions with locally univalent derivatives. Trans. Amer. Math. Soc., 1971, vol. 162, pp. 395-409.

[4] Chuaqui M., Duren P., Osgood B. The Schwarzian derivative for harmonic mappings. J. Anal. Math., 2003, vol. 91, pp. 329-351. DOI: 10.1007/BF02788793.

[5] Chuaqui M., Duren P., Osgood B. Univalence criteria for lifts of harmonic mappings to minimal surfaces. J. Geom. Anal., 2007, vol. 17, no. 1, pp. 49-74.

[6] Clunie J., Sheil-Small T. Harmonic univalent functions. Ann. Acad. Sci. Fenn. Math., 1984, vol. 9, pp. 3-25.

[7] Duren P. Univalent functions. N. Y.: Springer-Verlag, 1983, 395 p.

[8] Duren P. Harmonic mappings in the plane. Cambridge, 2004, 214 p.

[9] Gehring F. W., Pommerenke Ch. On the Nehari univalence criterion and quasicircles, Comment. Math. Helv., 1984, vol. 59, pp. 226-242.

[10] Godula J., Starkov V. V. On the sharpness of some inequalities of D. M. Campbell and Ch. Pommerenke. Math. Notes, 1998, vol. 68, no. 5, pp. 665-672 (in Russian).

[11] Golusin G. M. Geometrical function theory. Moscow, 1966, 628 p. (in Russian). 
[12] Graf S. Yu., Eyelangoli O. R. Differential inequalities in linear- and affineinvariant families of harmonic mappings. Russian Math. (Iz. VUZ), 2010, vol. 55, no. 10, pp. 60-62. DOI: 10.3103/S1066369X10100075.

[13] Graf S. Yu., Starkov V. V., Ponnusamy S. Radii of covering disks for locally univalent harmonic mappings. Monatsh. Math., 2016, vol. 180, no. 3, pp. 527-548. DOI: 10.1007/s00605-016-0904-2.

[14] Graf S. Yu. On the Schwarzian norm of harmonic mappings. Probl. Anal. Issues Anal., 2016, vol. 5 (23), no. 2, pp. 20-32. DOI: 10.15393/j3.art.2016.3511.

[15] Hernández R., Martín M. J. Pre-Schwarzian and Schwarzian derivatives of harmonic mappings. J. Geom. Anal., 2015, vol. 25 (1), pp. 64-91. DOI: 10.1007/s12220-013-9413-x.

[16] Hernández R., Martín M. J. Criteria for univalence and quasiconformal extension of harmonic mappings in terms of the Schwarzian derivative. Arch. Math., 2015, vol. 104, pp. 53-59. DOI 10.1007/s00013-014-0714-5.

[17] Hinton D. Sturms 1836 oscillation results evolution of the theory. SturmLiouville Theory, 2005, pp. 1-27. DOI: 10.1007/3-7643-7359-8_1.

[18] Nehari Z. The Schwarzian derivatives and schlicht functions. Bull. Amer. Math. Soc., 1949, vol. 55, no. 6, pp. 545-551.

[19] Nehari Z. Some criteria of univalence. Proc. Amer. Math. Soc., 1954, vol. 5 , pp. $700-704$.

[20] Pommerenke Ch. Linear-invariante Familien analytischer Functionen. I. Math. Ann., 1964, vol. 155 (2), pp. 108-154. DOI: 10.1007/BF01344077.

[21] Sheil-Small T. Constants for planar harmonic mappings. J. Lond. Math. Soc., 1990, vol. s2-42, pp. 237-248. DOI: 10.1112/jlms/s2-42.2.237.

[22] Sobczak-Knec M., Starkov V. V., Szynal J. Old and new order of linear invariant family of harmonic mappings and the bound for Jacobian. Ann. Univ. Mariae Curie-Sklodowska. Sect. A., 2011, vol. 65 (2), pp. 191-202.

Received September 13, $201 \%$.

In revised form, December 8, $201 \%$.

Accepted December 13, $201 \%$.

Published online December 14, $201 \%$.

Tver State University

33, Zheliabova st., Tver 170100, Russia;

Petrozavodsk State University

33, Lenina st., Petrozavodsk 185910, Russia

E-mail: Sergey.Graf@tversu.ru 